\title{
Intestinal bacteria induce TSLP to promote mutualistic T-cell responses
}

\author{
I Mosconi $^{1}$, MB Geuking ${ }^{2}$, MM Zaiss ${ }^{1}$, JC Massacand $^{1}$, C Aschwanden ${ }^{1}$, CKC Kwong Chung ${ }^{2}$, \\ KD $\mathrm{McCoy}^{2}$ and NL Harris ${ }^{1}$
}

\begin{abstract}
Thymic stromal lymphopoietin (TSLP) is constitutively expressed in the intestine and is known to regulate inflammation in models of colitis. We show that steady-state TSLP expression requires intestinal bacteria and has an important role in limiting the expansion of colonic Thelper type 17 (Th17) cells. Inappropriate expansion of the colonic Th17 cells occurred in response to an entirely benign intestinal microbiota, as determined following the colonization of germ-free C57BL/6 or TSLPR $^{-1-}$ mice with the altered Schaedler flora (ASF). TSLP-TSLPR (TSLP receptor) interactions also promoted the expansion of colonic Helios ${ }^{-}$Foxp $^{+}{ }^{+}$regulatory T cells, necessary for the control of inappropriate Th17 responses following ASF bacterial colonization. In summary, these data reveal an important role for TSLP-TSLPR signaling in promoting steady-state mutualistic T-cell responses following intestinal bacterial colonization.
\end{abstract}

\section{INTRODUCTION}

The human body harbors an immense population of harmless bacteria that colonize the gastrointestinal tract soon after birth to reach densities of $10^{11}$ or $10^{12}$ cells per g of luminal contents. Although we typically live in relative harmony with these organisms, it is now well recognized that intestinal homeostasis requires the active generation of mutualistic $\mathrm{T}$-cell responses constituted by a tightly regulated $\mathrm{CD} 4^{+} \mathrm{T}$-cell compartment that includes $\mathrm{T}$ helper type 1 (Th1), Th17, and Foxp3 ${ }^{+}$ regulatory T (Treg) cells. ${ }^{1,2}$ Th17 and Th1 cells are constitutively present in the healthy intestinal mucosa, and activated Foxp $3^{+}$Treg cells are expanded by the presence of intestinal bacteria., ${ }^{3,4}$ These bacterial-induced Treg are critical for preventing inappropriate or exaggerated activation of inflammatory Th1 and/or Th17 responses. ${ }^{3}$ Thus, by tightly regulating the different types of $\mathrm{CD} 4{ }^{+}{ }^{T}$-cell subsets present in the intestine, the host ensures the maintenance of immune homeostasis and thereby prevents detrimental bacterial dissemination from the intestinal lumen to sterile internal organs. ${ }^{5}$

Thymic stromal lymphopoietin (TSLP) is an epithelialderived cytokine that was originally isolated in 1994 from a mouse thymic stromal cell line $e^{6}$ and characterized as a lymphocyte growth factor. ${ }^{7,8}$ It is a 140 amino-acid four-helix bundle cytokine that is closely related to interleukin-7 (IL-7) with which it shares an overlapping but distinct biological profile. ${ }^{9}$ TSLP exerts its biological activities by binding to a heterodimeric receptor that consists of the IL-7 receptor $\alpha$ chain (IL-7R $\alpha$ ) and the TSLP receptor (TSLPR) chain, which is closely related to the common cytokine-receptor $\gamma$ chain $(\gamma \mathrm{c})$. TSLPR has low affinity for TSLP but in combination with IL-7R $\alpha$ generates a high-affinity-binding site for TSLP and triggers signaling through signal transducer and activator of transcription factor 5. ${ }^{9}$ TSLP is expressed predominantly by epithelial cells in the thymus, lung, skin, intestine, and tonsils, as well as stromal cells, mast cells, and dendritic cells (DCs). ${ }^{9,10}$ Expression of TSLP is regulated by nuclear factor- $\kappa \mathrm{B}$ and can be induced by exposure to viral, bacterial, or parasitic products, inflammatory cytokines, and the Th 2 cell-associated cytokines IL-4 and IL-13. ${ }^{11-16}$ TSLP has important roles in conditioning DCs to drive Th2 differentiation ${ }^{17}$ and its expression is increased in immunopathologies associated with dysregulated Th2 cell-type cytokine production, including atopic dermatitis $^{18}$ and asthma. ${ }^{19}$ It has additionally been reported to promote antibody isotype class switching in $\mathrm{B}$ cells, ${ }^{20}$ to regulate the development of Foxp $3^{+}$Tregs in the human thymus, ${ }^{19,21}$ and to promote basophil hematopoiesis. ${ }^{22}$

\footnotetext{
${ }^{1}$ Swiss Vaccine Research Institute and Global Health Institute, Ecole Polytechnique Fédérale de Lausanne (EPFL), Lausanne, Switzerland and ${ }^{2}$ Maurice Müller Laboratories, DKF, Universitätsklinik für Viszerale Chirurgie und Medizin, University Hospital (Inselspital), University of Bern, Bern, Switzerland. Correspondence: NL Harris (nicola.harris@epfl.ch)
}

Received 29 August 2012; revised 18 January 2013; accepted 5 February 2013; published online 20 March 2013. doi:10.1038/mi.2013.12 
In the intestine, TSLP has been reported to be constitutively expressed with the highest expression noted in the colon. ${ }^{11}$ It has a critical role in the regulation of the inflammatory responses following intestinal damage or helminth infection. ${ }^{10,11,23}$ Specifically, TSLP was reported to be necessary for protective Th2 immunity following Trichuris muris helminth infection ${ }^{11,23}$ as a result of its ability to limit infection-induced IL-12p40 production. ${ }^{23}$ Besides its important role in promoting Th2-dependent immune responses, growing evidence supports a role for TSLP in limiting Th1- and Th17-driven damage in the inflamed intestine. ${ }^{10,11}$ TSLPR-deficient specific pathogen-free (SPF) mice develop more severe inflammation and elevated Th1 and Th17 cytokines following dextran sodium sulfate (DSS)-mediated epithelial insult ${ }^{11}$ or in a model of $\mathrm{CD} 4{ }^{+}$ T-cell transfer colitis. ${ }^{10}$ Furthermore, TSLP is critical in mediating mucosal healing after DSS insult through its ability to stimulate secretory leukocyte peptidase inhibitor expression by intestinal epithelial cells (IECs). ${ }^{24}$

We addressed here whether TSLP has a role in regulating intestinal homeostasis under steady-state conditions. We show that TSLP is upregulated in the colon in response to colonization with intestinal bacteria. Bacterial-induced TSLP prevented excessive T-cell IL-17 and interferon- $\gamma$ (IFN $\gamma$ ) production, and the absence of TSLP-TSLPR signaling resulted in a notable hyperplasia of colonic lymphoid follicles (LFs). The dysregulated T-cell cytokine response occurred even in the presence of a limited and entirely benign bacterial community, the altered Schaedler flora (ASF), ${ }^{25}$ and was associated with a defective expansion of colonic Foxp $3^{+}$Helios ${ }^{-}$Treg cells upon colonization. These data position TSLP as a key cytokine necessary for the proper generation of mutualistic T-cell responses following colonization of the intestine with a benign bacterial community.

\section{RESULTS}

TSLP expression is elicited by intestinal bacteria and prevents excessive inflammatory cytokine production by T cells

TSLP is expressed by both IECs and intestinal DCs, ${ }^{10,11}$ and its expression within the intestine positively correlates with the density of luminal bacteria. ${ }^{11}$ Thus, to determine whether intestinal bacteria elicit intestinal TSLP expression, we compared TSLP mRNA levels across different sections of the small and large intestine in SPF mice (harboring a complex diversity of bacterial species) and germ-free mice (raised within a sterile environment with no exposure to intestinal bacteria). In keeping with the literature, TSLP expression was low throughout the small intestine and increased in the colon, peaking within the medial colonic section (Figure 1a). In contrast to increased TSLP expression in the colon of SPF mice, TSLP expression remained minimal throughout the intestine of germ-free mice, indicating that TSLP expression is upregulated following contact of the large intestinal epithelium with intestinal bacteria and/or their products (Figure 1a). We further addressed whether TSLP expression was dependent on the complexity of the commensal
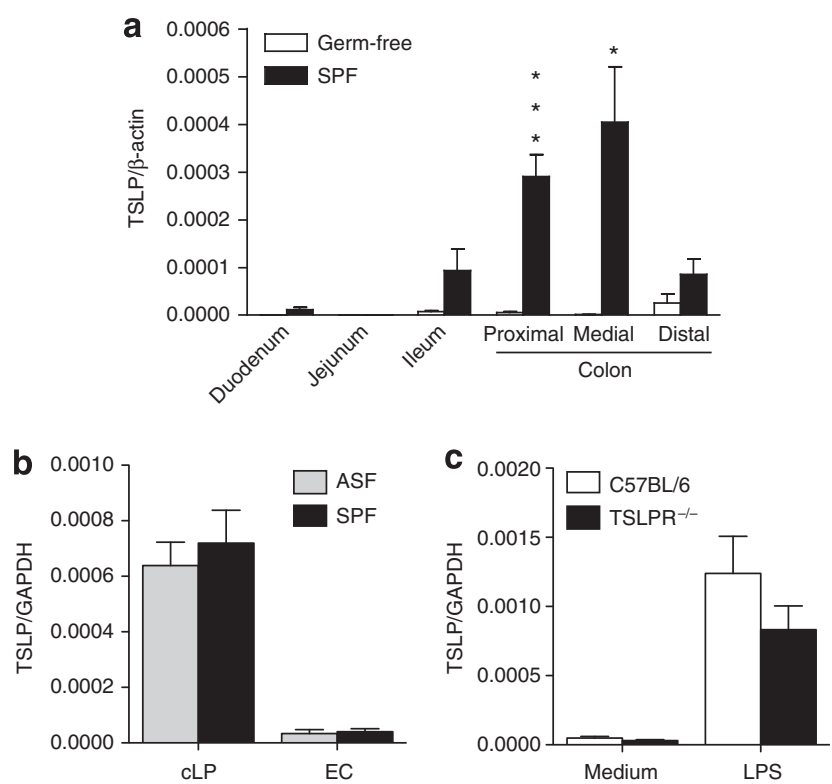

Figure 1 Thymic stromal lymphopoietin (TSLP) expression is induced by commensal bacteria in the colon. (a) Real-time reverse transcriptasePCR (RT-PCR) analysis of TSLP mRNA expression in freshly isolated tissues from germ-free or specific pathogen-free (SPF) mice. (b) Realtime RT-PCR analysis of TSLP mRNA expression in the epithelial cell (EC) or colonic lamina propria (cLP) fractions from altered Schaedler flora (ASF) and SPF. (c) Real-time RT-PCR analysis of TSLP mRNA expression in bone marrow-derived dendritic cells from C57BL/6 or TSLPR $^{-1-}$ mice. Results are normalized to $\beta$-actin or GAPDH (glyceraldehyde 3-phosphate dehydrogenase) and are shown from one representative experiment of two independent experiments $(n=4-5$ per group). LPS, lipopolysaccharide. ${ }^{\star} P<0.05$.

flora by analyzing mice colonized with a limited and entirely benign bacterial community, the ASF. ${ }^{25}$ As shown in Figure 1b, TSLP is also induced in ASF mice. As TSLP was reported to be expressed by both IECs and DCs, ${ }^{10,11}$ we next compared expression by IEC or lamina propria (LP) and found that while colonic IECs expressed TSLP (Figure 1b), most of the TSLP mRNA expression was derived from the LP fraction, most probably from DCs (Figure 1b). Similar results were also obtained from ASF mice (Figure 1b). Importantly, the absence of the TSLPR did not impact on TSLP expression by bone marrow-derived DCs, indicating that no positive feedback loop exists for this signaling pathway (Figure 1c). Taken together, these data showed that intestinal TSLP expression is induced by the commensal flora and is predominately produced by cells located within the LP.

To address whether TSLP expression impacted on intestinal homeostasis in the face of a complex intestinal bacterial community, we analyzed cytokine production by $\mathrm{T}$ cells present within the colonic lamina propria (cLP) of SPF TSLPR ${ }^{-1-}$ mice. Although the absence of TSLP-TSLPR signaling did not impact on the total number of $\mathrm{CD} 4^{+}$or $\mathrm{CD} 8^{+} \mathrm{T}$ cells present in the CLP, an increased proportion of $\mathrm{CD}^{+}{ }^{+} \mathrm{T}$ cells were observed to produce the inflammatory cytokine IL-17A (Figure 2a,c). Reproducible increases in the proportion of $\mathrm{CD} 4^{+} \mathrm{T}$ cells and $\mathrm{CD} 8{ }^{+} \mathrm{T}$ cells producing IFN $\gamma$ could also be observed, although these never reached statistical significance 


\section{a}
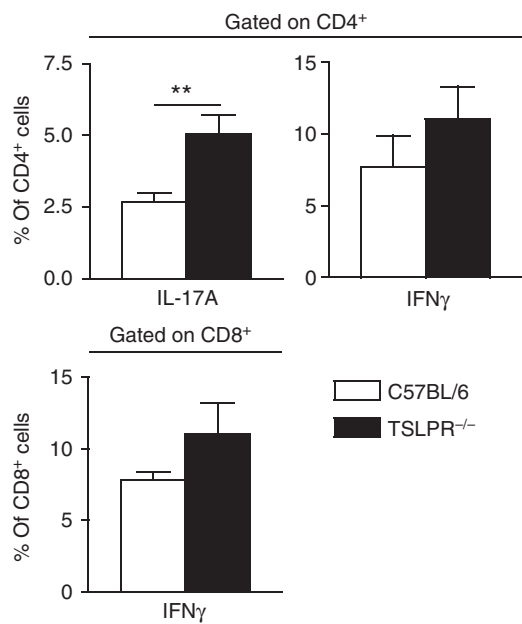

b
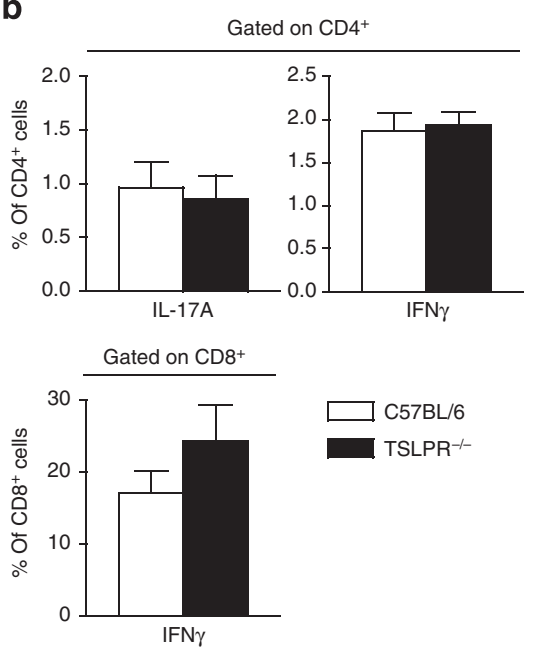

C
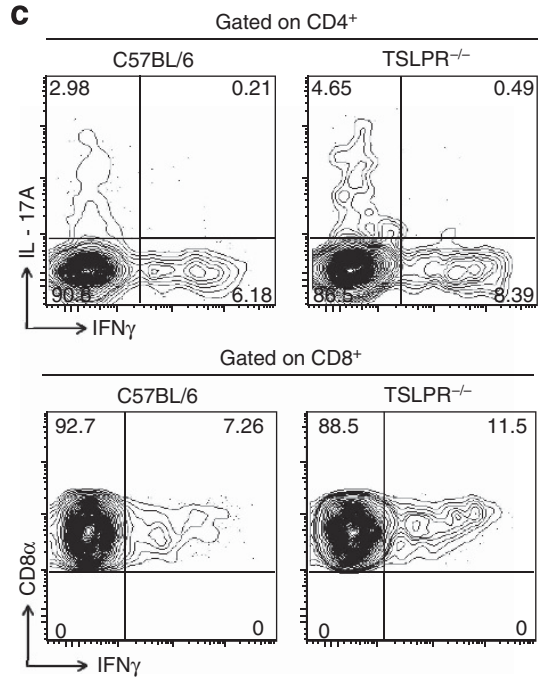

d
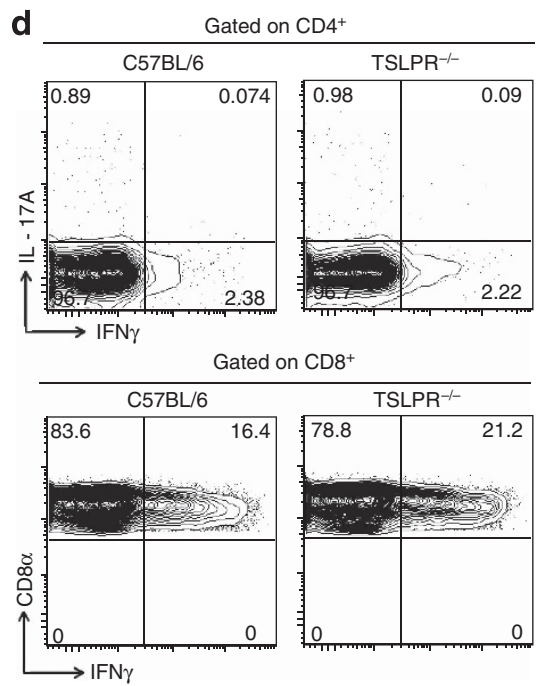

Figure 2 Thymic stromal lymphopoietin (TSLP) expression regulates T-cell inflammatory cytokine production. Quantification and representative plots of interleukin-17A (IL-17A) and interferon- $\gamma(\mathrm{IFN} \gamma)$ production by $\mathrm{CD}^{+}{ }^{+}$and $\mathrm{CD} 8^{+}$cells isolated from $(\mathbf{a}, \mathbf{c})$ colonic lamina propria or $(\mathbf{b}, \mathbf{d})$ mesenteric lymph nodes of naive specific pathogen-free mice after phorbol 12-myristate 13-acetate/lonomycin restimulation. Results are pooled from two independent experiments ( $n=3-4$ per group per experiment). ${ }^{* \star} P<0.01$.

(Figure 2a,c). By contrast, no differences in the proportion of $\mathrm{CD}^{+}$or $\mathrm{CD}^{+}{ }^{+} \mathrm{T}$ cells producing IL-17A or IFN $\gamma$ was observed in the mesenteric lymph nodes (MLN) of wild-type C57BL/6 or TSLPR ${ }^{-1-}$ animals (Figure $2 \mathbf{b}, \mathbf{d}$ ).

TSLP-TSLPR signaling does not impact on the number or phenotype of antigen-presenting cells (APC) present in the colon

DCs and macrophages (MPs) are important for immunological homeostasis in the colon with specific subsets known to represent regulatory or pro-inflammatory cell types. ${ }^{26}$ To determine whether TSLPR deficiency also resulted in alterations to APCs, we compared the number of cLP MPs and DCs in $\mathrm{C} 57 \mathrm{BL} / 6$ or TSLPR ${ }^{-/-}$animals. MPs were identified as a MHC (major histocompatibility complex) II $^{\text {high }} \mathrm{F} 4 / 80^{\text {high- }}$ CD11 $c^{\text {intermediate }}$ population, whereas DCs were identified as MHC II ${ }^{\text {high }} \mathrm{F} 4 / 80^{\text {intermediate }} \mathrm{CD} 11 \mathrm{c}^{\text {high }}$ population. DCs were then further divided into three subsets according to CD11b and CD103 expression (Figure 3a). Previous work identifying colonic APCs using a similar approach indicated that the MPs represent a regulatory IL-10-producing population. ${ }^{26}$ The $\mathrm{CD}_{11 \mathrm{~b}}{ }^{+} \mathrm{CD}_{103}{ }^{+} \mathrm{DC}$ population (represented here by the R2 DCs) is thought to represent migratory cells that can act as potent APCs and are characterized by the ability of producing retinoic acid and transforming growth factor- $\beta$ and inducing $d e$ novo differentiation of Tregs. ${ }^{27-30}$ The CD11b ${ }^{-} \mathrm{CD}_{103}{ }^{+} \mathrm{DC}$ population (R3) is thought to represent DCs located in intesitnal lymphoid tissues, most probably the isolated lymphoid follicles (ILFs), ${ }^{29}$ while the $\mathrm{CD}_{11 \mathrm{~b}}{ }^{+} \mathrm{CD} 103^{-}$ DCs (R1) represent more controversial subset that have been classified either as DCs or as MPs based on their apparent nonmigratory nature and poor antigen-presenting capacity. ${ }^{27-29}$ In our study, no major impact of TSLPR deficiency on the proportion of each investigated subpopulation was noted 
a

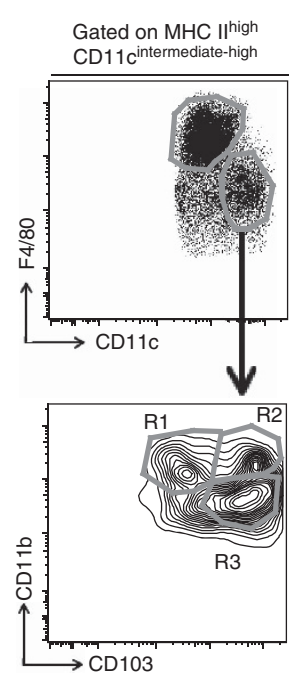

b

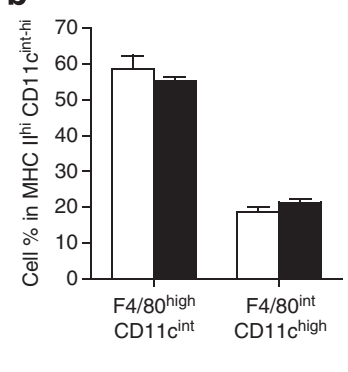

\section{C}
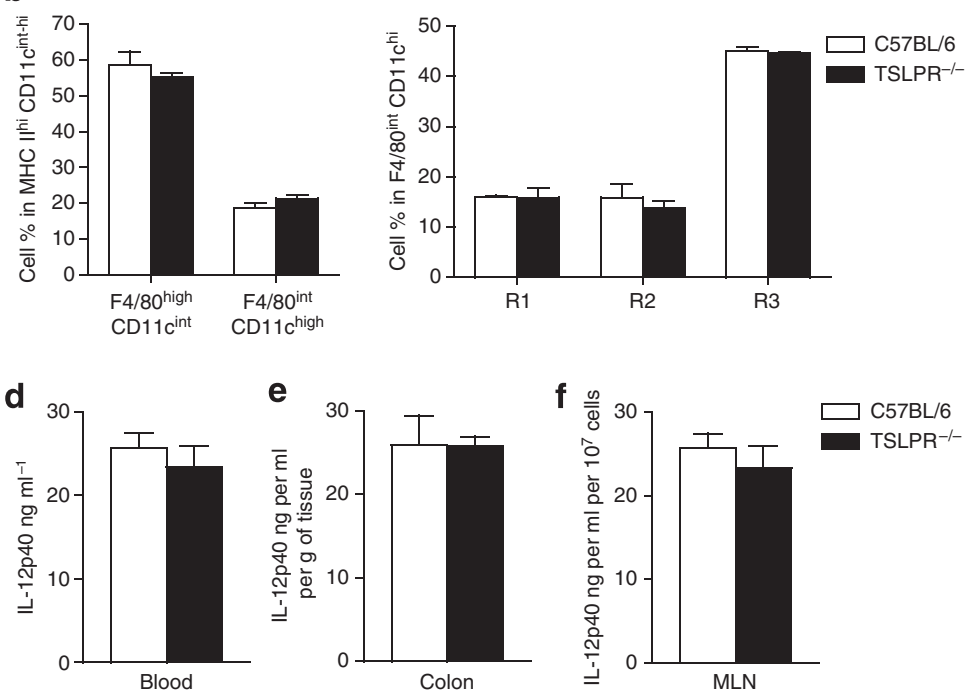

Figure 3 Thymic stromal lymphopoietin receptor (TSLPR) deficiency does not impact on the development or function of intestinal antigen-presenting cell subsets. (a) Gating strategy to identify colonic macrophages and dendritic cell (DC) subsets. Percentages of (b) total macrophages and DCs and (c) DC subsets in the colonic lamina propria of specific pathogen-free (SPF) TSLPR ${ }^{-1}$ and C57BL/6 mice. Data are pooled from two independent experiments ( $n=4-5$ per group per experiment). Interleukin-12p40 (IL-12p40) concentration in the (d) serum, (e) colon or (f) mesenteric lymph nodes (MLN) culture supernatants of naive SPF C57BL/6 and TSLPR ${ }^{-1-}$ mice. Results are shown from one experiment $(n=4-5$ per group) and are representative of three independent experiments. MHC, major histocompatibility complex.

(Figure $\mathbf{3 b}, \mathbf{c}$ ), indicating that this cytokine does not alter the development of either colonic DCs or MPs. To further assess whether TSLPR signaling had any impact on the activation status of these cell subsets, we determined the expression of the activation molecules CD40, CD80, and CD86. No differences were found in the expression of these molecules in TSLPR ${ }^{-1-}$ mice compared with wild-type mice (see Supplementary Figure S1 online).

IL-12p40 is a master regulator of Th1/Th17 differentiation, ${ }^{31}$ and TSLP can limit lipopolysaccharide or helminth-elicited IL-12p40 production by DCs. ${ }^{11,23,32}$ We therefore determined whether TSLPR signaling impacted on the steady-sate production of IL-12p40 in the cLP in response to intestinal bacterial. Analysis of cytokine levels in colon explants, mesenteric lymph node cells, and serum of SPF mice revealed no difference in basal IL-12p40 production in the presence or absence of TSLPR signaling (Figure 2d-f).

Taken together, these data indicate that TSLP-TSLPR signaling does not impact on the number or functional status of major APC subsets resident in the intestinal colon under steady-state conditions.

\section{TSLPR-deficient mice exhibit hypertrophic development of colonic LFs}

We next assessed whether the changes in effector T-cell cytokine production (shown in Figure 2) impacted on the overall structure of the colon. In agreement with previous reports, ${ }^{11}$ we observed no major histological alterations to the gross colonic architecture of naive $\mathrm{TSLPR}^{-1-}$ mice (Figure 4a). However, we did note a significant increase in the number (Figure $4 \mathbf{b}$ ) and size (Figure 4c,d ) of mature LFs in TSLPR $^{-1-}$ mice. Notably, in germ-free mice, we did not observe any significant difference in the occurrence or size of mature LF in the colon, indicating that the observed phenotype requires interactions with the intestinal bacterial flora (see Supplementary Figure S2 online). B-cell-rich follicles found within the anterior small intestine and colon can be represented by ILFs formed in response to stimuli derived from luminal Gram-negative bacteria ${ }^{33}$ or tertiary lymphoid follicles (tLFs) formed in response to chronic inflammation. ${ }^{34}$ Hyperplasia of intestinal LFs has previously been reported for mice exhibiting aberrant production of secretory immunoglobulin $\mathrm{A}(\mathrm{sIgA}),{ }^{35}$ lacking Peyer's Patches ${ }^{36}$ or following DSS administration. ${ }^{34}$ We therefore checked whether the higher proportion of inflammatory $\mathrm{T}$ cells and the increased numbers of LF in the TSLPR ${ }^{-1-}$ mice resulted from alterations in the intestinal microbiota community or an inability to contain the microbiota due to defective humoral immunity. Neither impact of TSLPR signaling was noted for the major bacterial phyla present within the intestine of naive mice (either Gram -ve or Gram + ve) nor were any defects in sIgA production observed (see Supplementary Figure S3 online). These data are consistent with previous reports ${ }^{24}$ and indicate that the enlarged LFs present in TSLPR ${ }^{-1-}$ mice may represent tLFs that developed in response to the dysregulated T-cell response and chronically elevated levels of inflammatory cytokines.

\section{TSLP-TSLPR signaling limits the development of inflammatory $\mathrm{CD}^{+}{ }^{+} \mathrm{T}$-cell responses following colonization of germ-free mice with a minimal intestinal microbiota}

To determine whether the exaggerated inflammatory cytokine production by colonic $\mathrm{T}$ cells in TSLPR ${ }^{-1-}$ mice occurred in response to luminal intestinal bacteria, and to rule out the 
a
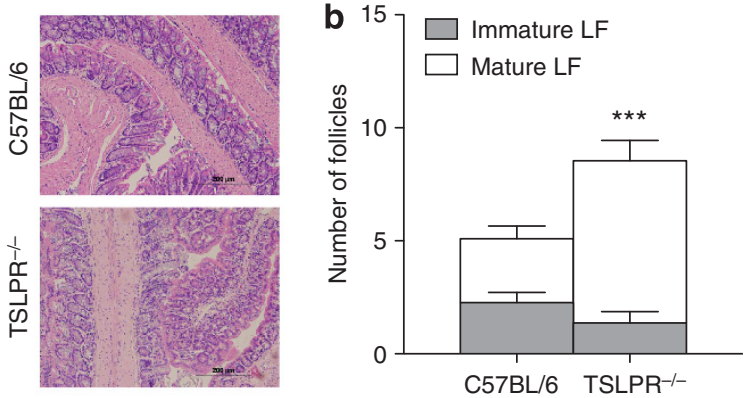

d

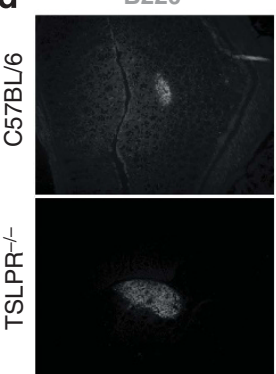

$\lg A$

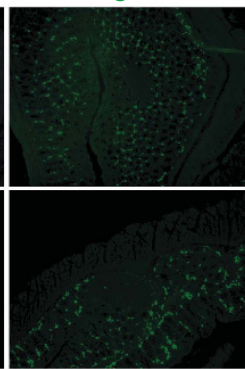

CD11C

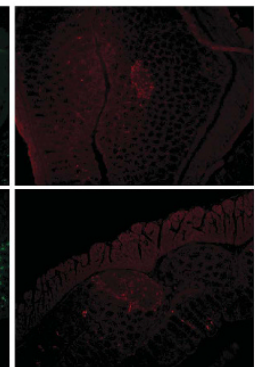

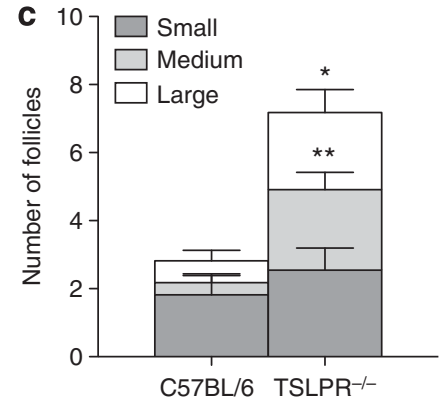

DAPI

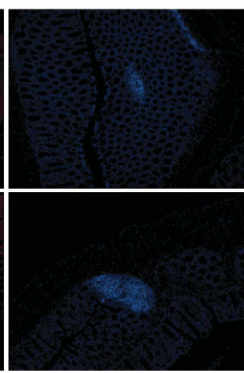

Merged

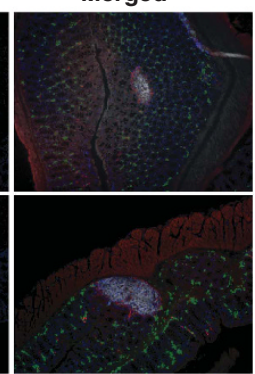

Figure 4 Thymic stromal lymphopoietin (TSLP)-deficient mice exhibit a hyperplasia of colonic lymphoid follicles (LFs). (a) Histological sections from the colon of naive specific pathogen-free (SPF) C57BL/6 and TSLP receptor (TSLPR) ${ }^{-1}$ - mice. Quantification of the (b) number of immature and mature LFs and (c) size of mature LFs in the same mice. (d) Representative microscopic picture of a mature LF from naive SPF C57BL/6 and TSLPR ${ }^{-1-}$ mice. Data are from one representative experiment of two seperate experiments ( $n=3$ per group). DAPI, 4,6-diamidino-2-phenylindole; IgA, immunoglobulin A. ${ }^{\star} P<0.05,{ }^{* \star} P<0.01,{ }^{* * \star} P<0.001$.

possibility of a pathogenic contaminant, we re-derived this strain to germ-free conditions and performed experiments analyzing the response of colonic $\mathrm{T}$ cells to colonization with the defined ASF. In keeping with previous observations, ${ }^{3}$ colonization of wild-type C57BL/6 mice with the benign ASF flora did not significantly alter the percentage of effector CD4 ${ }^{+}$ T cells secreting IL-17A or IFN $\gamma$ in the cLP or MLN (Figure 5). By contrast, ASF colonization of TSLPR ${ }^{-/}$mice resulted in an aberrant IL-17A and IFN $\gamma$ response in both the organs (Figure 5), which is in line with our observations in SPF mice (Figure 2).

\section{TSLP-TSLPR signaling promotes the expansion of Foxp $3^{+}$Helios ${ }^{-}$Treg cells within the colon}

Our earlier experiments showed that altered inflammatory T-cell responses in TSLPR ${ }^{-1-}$ mice were not due to gross changes in local APC populations (Figure 3 and see Supplementary Figure S1 online), IL-12p40 production (Figure 2), and protective sIgA or intestinal bacterial communities (see Supplementary Figure S3 online). We therefore hypothesized that the dysregulated effector $\mathrm{T}$-cell responses may arise as a consequence of altered Treg cell function. Helios, an Ikaros transcription factor family member, has been proposed to differentiate thymic-derived from peripherally induced Foxp ${ }^{+}$Treg cells, ${ }^{37}$ although the exact role that Helios has in Treg development remains unclear. Of interest to the current study, it has previously been shown that the majority of Tregs present in the colon of germ-free mice are Helios ${ }^{+}$and that bacterial colonization induces a conversion and/or expansion of the Helios ${ }^{-}$Treg population. ${ }^{3,4,38}$
Moreover, bacterial-induced expansion of colonic Foxp3 ${ }^{+}$ Helios $^{-}$Treg cells has an essential role in preventing exaggerated Th1 and Th17 responses and is necessary to promote the successful establishment of intestinal homeostasis. $^{3}$ Examination of the Foxp ${ }^{+}$T-cell compartment in TSLPR $^{-/-}$mice showed that TSLP-TSLPR signaling was necessary for the efficient generation of Foxp $3^{+}$Helios ${ }^{-}$Treg cells in cLP following ASF colonization (Figure 5). This observed ability of TSLPR signaling to regulate colonic Treg cell phenotype is likely to be responsible for the expansion of Th17 cells in TSLPR ${ }^{-1-}$ mice. By contrast, no differences in the Helios ${ }^{-}$Treg population were observed in SPF mice in the cLP (see Supplementary Figure S4A online) or MLN (see Supplementary Figure S4B online) probably as a result of the more complex bacterial community masking the impact of TSLP on Treg generation in these mice. Lastly, both the exaggerated inflammatory cytokine production (Figure 5) and the decreased expansion of Foxp $3^{+}$Helios ${ }^{-}$Treg cells (Figure 6) observed in ASF-colonized $\mathrm{TSLPR}^{-/-}$mice could be replicated following treatment of wild-type C57BL/6J mice with a neutralizing TSLP (clone 28F12) monoclonal antibody throughout the period of ASF colonization (see Supplementary Figure S5 online).

\section{TSLP impacts directly on T cells in response to bacterial colonization}

We next investigated the expression levels of IL-7R $\alpha$ chain and TSLPR chain on FoxP3 ${ }^{-}$and FoxP $3{ }^{+} \mathrm{CD} 4{ }^{+} \mathrm{T}$ cells in the cLP and MLN. As shown in Figure 7, both FoxP3 ${ }^{-}$and FoxP3 ${ }^{+}$ $\mathrm{CD} 4{ }^{+} \mathrm{T}$ cells at these sites express a functional receptor for 
a
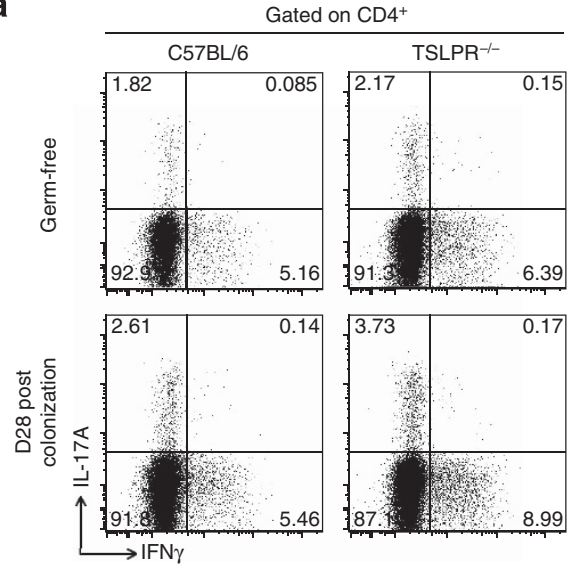

C
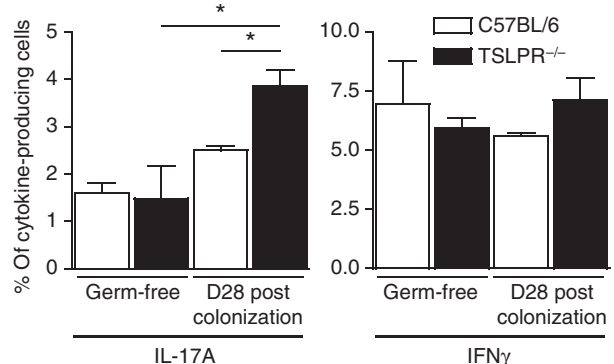

b
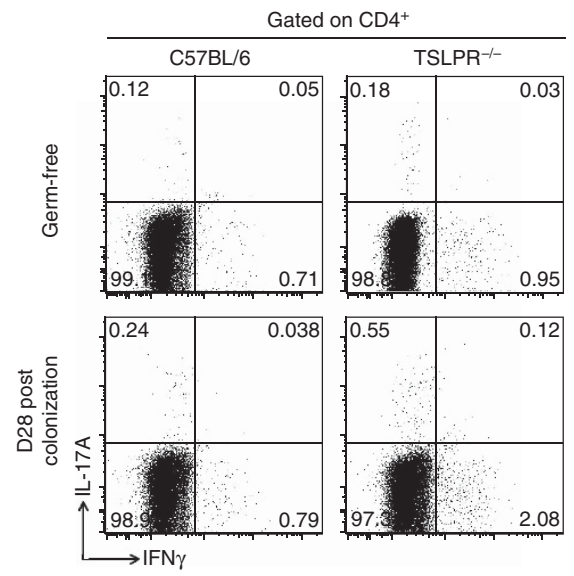

d
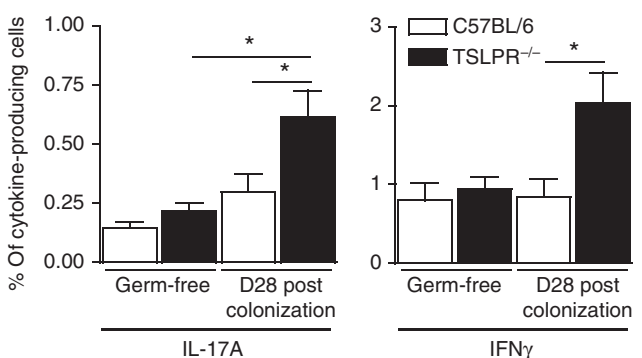

Figure 5 Interleukin-17A (IL-17A) production is upregulated in thymic stromal lymphopoietin receptor (TSLPR)-deficient germ-free mice following recolonization with altered Schaedler flora (ASF) bacteria. Representative plots and quantification of IL-17A and interferon- $\gamma$ (IFN $\gamma$ ) production by CD4 ${ }^{+}$ cells isolated from $(\mathbf{a}, \mathbf{c})$ colonic lamina propria (cLP) and $(\mathbf{b}, \mathbf{f})$ mesenteric lymph nodes (MLN) of germ-free and day 28 ASF-colonized mice following phorbol 12-myristate 13-acetate /lonomycin restimulation. Results are pooled from three independent experiments for the cLP ( $n=3-4$, per group per experiment) and are representative of two independent experiments for the MLN ( $n=3-4$ per group). ${ }^{*} P<0.05$.

TSLPR, raising the possibility that TSLP acts directly on these cells to modulate homeostasis. In order to determine whether TSLP can act directly on intestinal T cells, we transferred CD4 ${ }^{+}$ splenocytes from wild-type CD45.1 ${ }^{+}$congenic or TSLPR ${ }^{-1-}$ $\left(\mathrm{CD} 45.2^{+}\right)$donor mice (1:1 ratio) into germ-free $\mathrm{RAG}^{-1-}$ recipient mice. Colonization with ASF was started on the day of T-cell transfer. Strikingly, after 28 days, TSLPR ${ }^{-1-}$ Tregs $\left(\mathrm{CD} 45.2^{+}\right)$proliferated significantly less than wild-type Treg cells $\left(\mathrm{CD} 45.2^{-}\right)$both in the CLP and MLN (Figure 7b-d), thus implicating a direct effect of TSLP on homeostatic Treg proliferation in the intestinal compartment following colonization. In addition, increased IL-17A and IFN $\gamma$ production from TSLPR ${ }^{-1-}$ cells was detected in the MLN (see Supplementary Figure S6 online), indicating that TSLP may act on both Tregs and effector $T$ cells to promote successful immune adaptation in response to colonization.

\section{DISCUSSION}

TSLP has recently been shown to have a crucial role in negating overt inflammation in models of pathological colitis. ${ }^{10,11}$ Yet, it has remained unclear whether TSLP also regulates immune reactivity in the healthy steady state in response to colonization with commensal bacteria. In the current study, we investigated the role of TSLP-TSLPR signaling in the maintenance of intestinal homeostasis in mice harboring a complex intestinal microbiota (SPF mice) or following colonization of germ-free mice with a limited, and entirely benign, intestinal microbiota (ASF mice) to reduce the possibility of measuring potential pathobiont-mediated effects. Induction and expansion of colonic Treg cells following ASF colonization of germ-free mice is essential for the maintenance of immune homeostasis and prevents immune deviation exemplified by exaggerated Th1 and Th17 responses. ${ }^{3}$ We report a crucial role for the TSLP-TSLPR signaling pathway in mediating intestinal immune homeostasis. Disruption of this pathway, either by genetic TSLPR deficiency or by experimental in vivo TSLP neutralization, resulted in defective expansion of colonic Foxp $3^{+}$Helios ${ }^{-}$Treg cells and a consequent expansion of Th17 cytokine-producing $\mathrm{CD}^{+}{ }^{+} \mathrm{T}$ cells. Although still controversial, Helios ${ }^{-}$Tregs are thought to potentially represent bacteria-specific peripherally generated adaptive Treg while Helios ${ }^{+}$Treg, which do not seem to be affected by TSLPR deficiency, might represent the more self-reactive thymus-derived natural Treg population.,

It remains unclear whether disruption of immune homeostasis resulted from defective TSLPR signaling directly on $\mathrm{CD} 4{ }^{+} \mathrm{T}$ cells themselves or on innate intestinal cells such as DCs or both. The fact that we did not note an imbalance in major colonic APC populations or in intestinal IL-12p40 production indicates that TSLP may act directly on the T-cell compartment. This view is supported by the expression of a functional TSLPR on both FoxP3 ${ }^{-}$and FoxP $3^{+} \mathrm{CD} 4{ }^{+} \mathrm{T}$ cells 


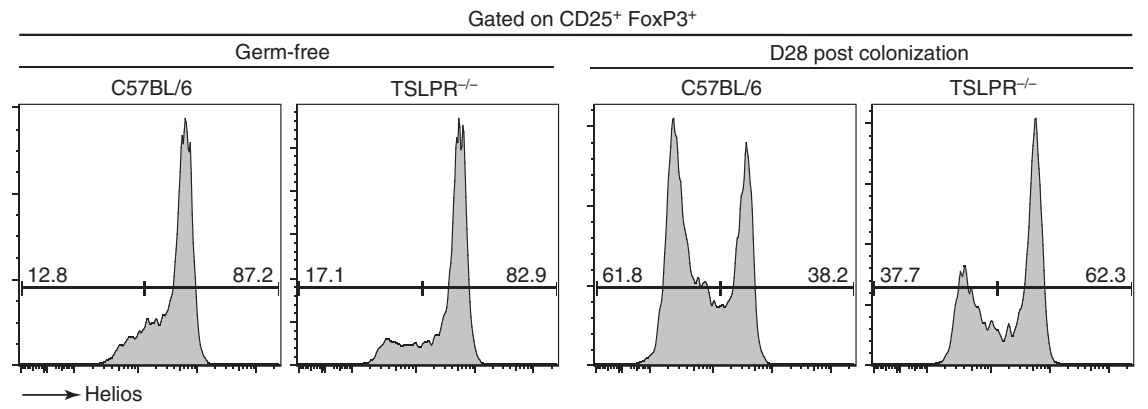

b
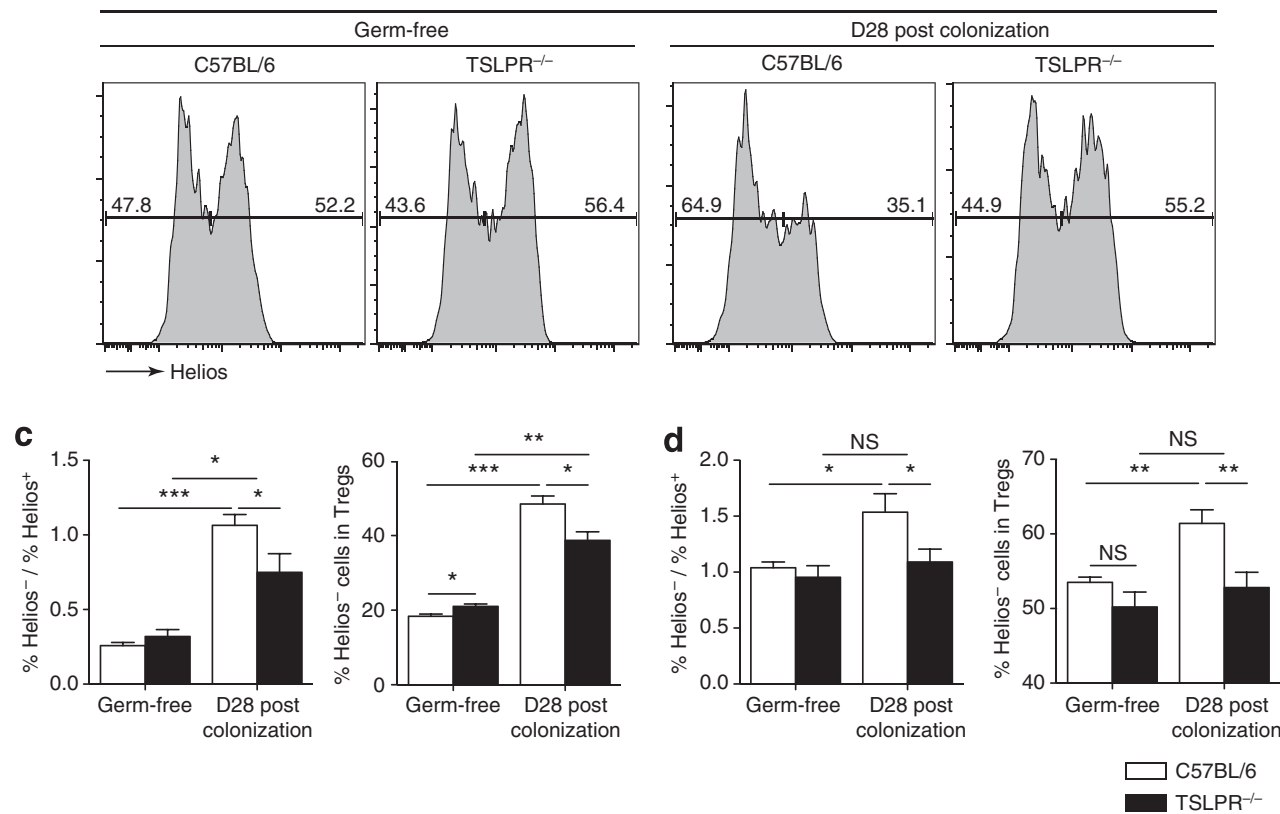

Figure 6 Thymic stromal lymphopoietin receptor (TSLPR) deficiency results in a failure to expand Foxp $3^{+}$Helios ${ }^{-}$colonic regulatory $\mathrm{T}(\mathrm{Treg})$ cells in response to colonization with altered Schaedler flora (ASF) bacteria. Representative histograms and quantification of $\mathrm{Helios}^{-} / \mathrm{Helios}^{+}$ratios in $^{-}$ $\mathrm{CD}^{2} 5^{+} \mathrm{FoxP}^{+}$cells from (a, c) colonic lamina propria (cLP) and (b, d) mesenteric lymph nodes (MLN) of germ-free and day 28 ASF-colonized mice. Results are pooled from three independent experiments for the cLP ( $n=3-4$, per group per experiment) and are representative of two independent experiments for the MLN ( $n=3-4$ per group). ${ }^{\star} P<0.05$, ${ }^{\star \star} P<0.01$, ${ }^{\star * \star} P<0.001$.

in the CLP and MLN and by the recent findings of Spadoni et al. ${ }^{10}$ who reported that TSLP functions to prevent severe colitis through a direct action on T cells. In support of our own findings, these authors reported a role for DC-derived TSLP in reducing the capacity of in vitro activated $\mathrm{CD}^{+}{ }^{+} \mathrm{T}$ cells to produce IL-17, while fostering the development of Foxp $3^{+}$ Treg cells. Furthermore, when we transferred TSLPR ${ }^{-1-}$ and wild-type $\left(\mathrm{CD} 45.1^{+}\right) \mathrm{CD} 4^{+} \mathrm{T}$ cells into $\mathrm{RAG}^{-1-}$ recipient mice, we noted that an absence of TSLPR signaling directly to $\mathrm{T}$ cells led to a reduced expansion of Tregs and an increased production of IL-17A and IFN $\gamma$.

TSLP has been previously reported to be expressed by colonic epithelial cells or LP DCs in response to bacterial stimulation in vitro. ${ }^{10,12,39}$ Our study indicates that a LP cell population, probably DCs, represents the predominant source of TSLP production in vivo. Interestingly, TSLP expression required the presence of intestinal bacteria thus providing a feedback loop between the recognition of bacteria, and/or their products, and the regulation of immune homeostasis. TSLP expression by DCs is known to be Myd88 (myeloid differentiation primary response gene 88)-dependent ${ }^{10}$ and activation of colonic Treg cells in response to ASF colonization requires Myd88/Ticam signaling. ${ }^{3}$ Myd88-dependent TSLP expression may therefore provide a possible link between recognition of commensal bacteria and T-cell homeostasis.

Although TSLPR deficiency resulted in an exaggerated Th1/ Th17 response and reduced expansion of Foxp $3^{+}$Helios ${ }^{-}$ Treg cells, this immune deviation did not result in pathological changes to the intestine as determined by histological examination. Despite the absence of overt inflammation, immune deviation in TSLPR-deficient mice did correlate with a notable hyperplasia of colonic LFs. This hyperplasia was not observed in germ-free $\mathrm{TSLPR}^{-1-}$ mice, indicating that it occurs as part of a dysregulated response to commensal bacteria. Intestinal LFs are represented by scattered B-cell-rich structures and can be represented by the histologically 
a

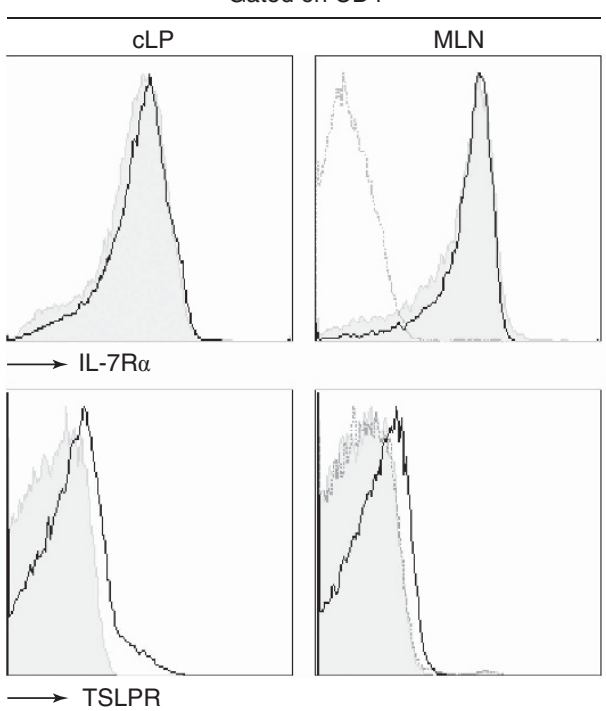

b
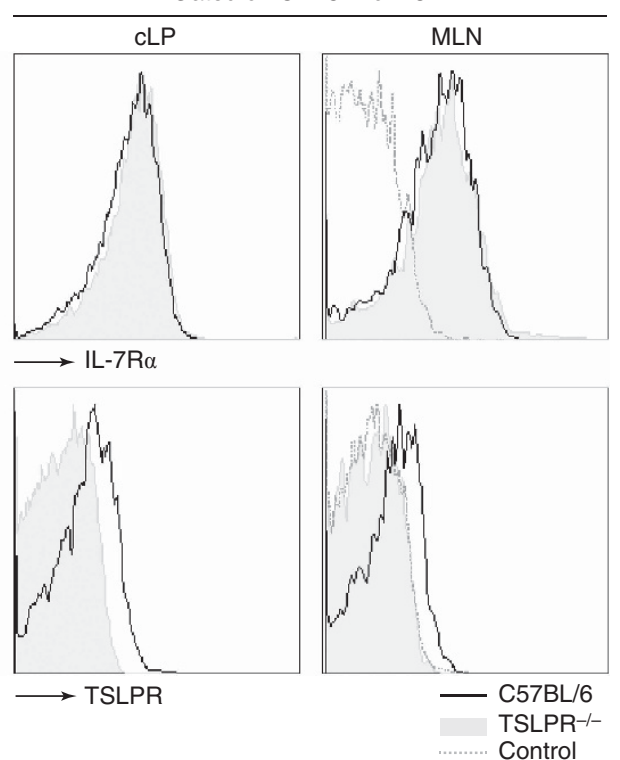

C

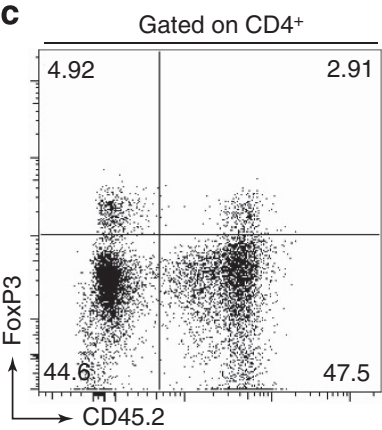

d

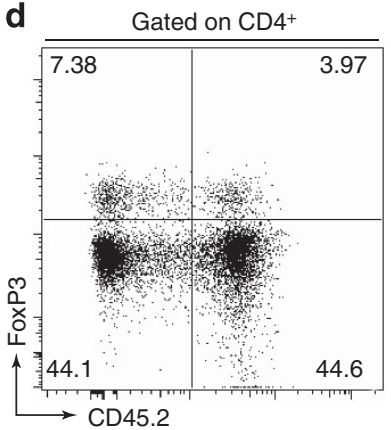

e

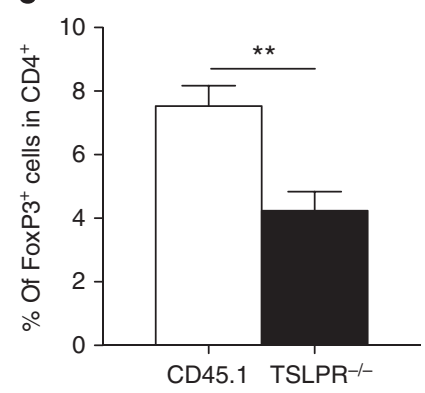

Figure $7 \mathrm{CD}^{+}{ }^{+} \mathrm{T}$ cells and regulatory $\mathrm{T}$ cells express the high-affinity receptor for thymic stromal lymphopoietin (TSLP) and respond directly to this cytokine. Representative histograms of interleukin-7R (IL-7R) and TSLP receptor (TSLPR) expression in (a) CD4 ${ }^{+}$and (b) CD4 $^{+}{ }^{+}$CD25 $5^{+}$FoxP3 ${ }^{+}$cells from colonic lamina propria (cLP) and mesenteric lymph nodes (MLN) of day 28 ASF-colonized C57BL/6 or TSLPR ${ }^{-1-}$ mice. Fluorescence Minus One Control (here indicated as control) was used for TSLPR and IL-7R $\alpha$ staining. Results are shown from one experiment and are representative of two independent experiments ( $n=3-4$ per group). Representative plots of TSLPR ${ }^{-1-}\left(\right.$ CD45.2 $\left.{ }^{+}\right)$and wild-type CD45.1 (CD45.2 $\left.{ }^{-}\right)$FoxP3 ${ }^{+}$cells isolated from (c) CLP and (d) MLN of day 28 ASF RAG ${ }^{-1-}$ mice reconstituted with CD4 ${ }^{+}$splenocytes from SPF TSLPR ${ }^{-1-}$ or wild-type CD45.1 mice. Quantification (e) of TSLPR ${ }^{-1-}\left(\mathrm{CD} 45.2^{+}\right)$and wild-type CD45.1 (CD45.2 ${ }^{-}$) FoxP3 ${ }^{+}$cells isolated from (c) cLP and (d) MLN. Results are shown from one experiment and are representative of two independent experiments $(n=5$, per group per experiment; mice were pooled for $c L P)$. ${ }^{\star \star} P<0.01$.

indistinguishable ILFs or tLFs. The development of ILFs is initiated by lymphoid tissue inducer cells present in cryptopatches. In this case, peptidoglycans shed from intestinal bacteria activate nucleotide-binding oligomerization domain containing 1 (NOD-1) in IECs and elicit the release of $\beta$-defensin-3 and C-C motif chemokine ligand 20, which activate cryptopatch lymphoid tissue inducer cells and B cells resulting in the accumulation lymphocytes and DCs to form ILFs. ${ }^{33}$ By contrast, formation of tLFs occurs in response to inflammation and is independent of lymphoid tissue inducer cells. Instead chemokines, such as $\mathrm{C}-\mathrm{X}-\mathrm{C}$ motif chemokine ligand 13, are released in response to inflammation and recruit activated lymphotoxin-expressing lymphocytes that interact with lymphotoxin beta receptor-expressing stromal cells to further augment chemokine production and adhesion molecule expression, resulting in a self-sustaining cellular cluster and the formation of an organized lymphoid tissue. ${ }^{40}$ Known modulators of ILF formation include bacterial peptidoglycans and SIgA. However, TSLP-TSLPR signaling was not found to impact on bacterial diversity or SIgA production. In contrast to our data, TSLP has previously been reported to promote IgA2 production in humans, ${ }^{20}$ thus TSLP-mediated regulation of IgA class switch recombination may represent a crucial difference between the murine and human cytokine.

Although we did not determine the exact nature of the LF present in TSLPR ${ }^{-1-}$ mice, the exaggerated production of T-cell-derived inflammatory cytokines suggests that the observed LFs may represent tLFs. Lipopolysaccharide-elicited T-cell-derived IL-17 production has recently been reported to promote chemokine secretion and tertiary lymphoid tissue development in the lung. ${ }^{41}$ Thus exaggerated and chronic IL-17A production may therefore provide a possible explanation for intestinal LF hyperplasia in TSLPR ${ }^{-1-}$ mice. As mentioned, TSLP is known to attenuate DSS-induced colitis. ${ }^{11}$ 
Interestingly, Lochner et al. ${ }^{34}$ reported that a hyperplasia of tertiary lymphoid tissues in the intestine predisposed mice to aggravated pathology following DSS administration. Thus increased tLF present in TSLPR-deficient mice may provide one explanation for the increased susceptibility of these animals to pathological colitis. Alternatively, increased colitis may occur as a direct result of the ability of TSLP to regulate T-cell immune deviation. Of note, pathological colitis has previously been associated with reduced TSLP expression in both mice ${ }^{10}$ and humans. ${ }^{32,42}$

In conclusion, we have shown that TSLP has a crucial role in the regulation of intestinal immune homeostasis. The absence of TSLP-TSLPR signaling results in immune deviation with exaggerated Th1/Th17 responses and reduced Treg cell activation even in the presence of a limited and benign bacterial community. This study provides the first proof of a role for TSLP in regulating baseline intestinal homeostasis as opposed to inflammatory colitis in vivo and lends support to previous findings associating defective TSLP expression with increased disease severity in Crohn's patients. ${ }^{32}$

\section{METHODS}

Mice and treatments. $\mathrm{C} 57 \mathrm{BL} / 6 \mathrm{~J}$ and $\mathrm{C} 57 \mathrm{BL} / 6 \mathrm{~J} \mathrm{TSLPR}^{-/-43}$ mice were bred and maintained at the EPFL animal facility under specific pathogen-free conditions. TSLPR ${ }^{-1-}$ mice were backcrossed to C57BL/6 mice for more than nine generations. Before each experiment, beddings were mixed between all the experimental groups for at least 3-4 weeks. Germ-free C57BL/6 and TSLPR ${ }^{-1-}$ mice were bred and maintained in flexible film isolators at the Clean Mouse Facility of the University of Bern and the Inselspital (Bern, Switzerland). Germ-free status was routinely confirmed by aerobic and anaerobic culture as well as sytox green (Invitrogen, Gaithersburg, MD) and Gram staining (Harleco, EMD Millipore Corporation, Billerica, MA) of cecal contents to detect unculturable organisms. ASF colonization was performed by placing an ASF-colonized mouse into a cage of germ-free animals. Animal experiments were performed according to the institutional guidelines and to Swiss federal and cantonal laws on animal protection. For in vivo neutralization of TSLP, C57BL/6J mice were injected with $200 \mu \mathrm{g}$ of TSLP-neutralizing antibody (clone 28F12) via the intraperitoneal route twice per week for 4 weeks starting from the day of colonization. Control mice were injected with Rat IgG2a isotype control (clone 2A3, BioXcell, West Lebanon, NH). The 28F12 hybridoma developed by Dr Andrew Farr was obtained from the Developmental Studies Hybridoma Bank developed under the auspices of the NICHD and maintained by the University of Iowa, Department of Biological Sciences, Iowa City, IA, USA.

Real-time quantitative PCR. Total RNA was isolated from all intestinal specimens using TRI Reagent (Molecular Research Center, Cincinnati, $\mathrm{OH}$ ) and reverse transcribed using RevertAid Reverse Transcriptase (Fermentas, Thermo Fisher Scientific, Wohlen, Switzerland). Transcribed cDNA was used as a template for the PCR reaction. Expression was normalized according to the expression of the housekeeping gene $\beta$-actin or GAPDH (glyceraldehyde 3 -phosphate dehydrogenase). Sequences of primers used: $\beta$-actin, $5^{\prime}$-CTTTTCACGGTTGGCCTTAG- $3^{\prime}$ and $5^{\prime}$-CCCTGAAGTACCCC ATTGAAC- $3^{\prime}$; GAPDH, $5^{\prime}$-GGGTGTGAACCACGAGAAAT- $3^{\prime}$ and $5^{\prime}$-CCTTCCACAATGCCAAAGTT- $3^{\prime}$; and TSLP, 5' -AGAATTAAC CTGAGGTCCGGAT- ${ }^{\prime}$ and AGAGAAGCCCTCAATGACCA- ${ }^{\prime}$.

Isolation of IECs. IECs were isolated as reported by Kang et al. ${ }^{44}$ Briefly, colons were cut into $2-3-\mathrm{mm}$ fragments and washed by phosphate-buffered saline five times with vigorous shaking. Then, intestines were incubated with $3 \mathrm{~mm}$ EDTA and $0.5 \mathrm{~mm}$ dithiothreitol at room temperature for $30 \mathrm{~min}$ with shaking.

Generation of bone marrow-derived DCs. Bone marrow cells were isolated from naive $\mathrm{C} 57 \mathrm{BL} / 6$ or TSLPR ${ }^{-1-}$ mice and bone marrowderived DCs were generated as described by Lutz et al. ${ }^{45}$ At day 10, bone marrow-derived DCs were collected and plated out at a concentration of $10^{5}$ cells per well in a 96-well round-bottomed plate. Cells were stimulated with complete medium (IMDM (Iscove's Modified Dulbecco's Medium; BioWhittaker, Lonza, Verviers, Belgium) containing 7\% fetal calf serum, L-glutamine, HEPES (4-(2-hydroxyethyl)1-piperazineethanesulfonic acid), $100 \mathrm{U} \mathrm{ml}^{-1}$ penicillin, and $100 \mu \mathrm{g} \mathrm{ml}^{-1}$ streptomycin and $25 \mu \mathrm{M}$ 2-mercaptoethanol (SigmaAldrich, Taufkirchen, Germany)) with or without $1 \mu \mathrm{g} \mathrm{ml}^{-1}$ lipopolysaccharide (from Escherichia coli 0111:B4; Sigma-Aldrich) for $3 \mathrm{~h}$.

Mononuclear cell isolation from cLP and MLN. cLP cells were isolated as previously described. ${ }^{3}$ Briefly, the colon was flushed, opened longitudinally, washed thoroughly in $\mathrm{Mg}_{2} \mathrm{Cl}_{2}$ - and $\mathrm{CaCl}_{2}$-free DPBS (Dulbecco's phosphate-buffered saline; GIBCO, Invitrogen). The organs were then cut into $3-5 \mathrm{~mm}$ pieces that were incubated $4-5$ times in $25 \mathrm{ml}$ EDTA/HEPES/DPBS solution at $37^{\circ} \mathrm{C}$ for $20 \mathrm{~min}$ in a shaking incubator in order to remove the epithelial layer. Intestinal pieces were collagenase-digested for $40 \mathrm{~min}$ at $37^{\circ} \mathrm{C}$ in $25 \mathrm{ml}$ IMDM containing $0.5 \mathrm{mg} \mathrm{ml}^{-1}$ collagenase type VIII (Sigma-Aldrich), $50 \mathrm{U}$ DNaseI (Roche Diagnostic, Nutley, NJ), and $0.01 \mathrm{~m}$ HEPES (GIBCO, Invitrogen). The crude cell suspension was loaded onto a $30 \% / 100 \%$ percoll (GE Healthcare, Milan, Italy) gradient and centrifuged at $680 \mathrm{~g}$ for $30 \mathrm{~min}$ at room temperature with the acceleration and brake turned off. Cells were collected from the $30 \% / 100 \%$ interphase. MLN were excised and incubated for $10 \mathrm{~min}$ at $37^{\circ} \mathrm{C}$ with Liberase TL (Roche Diagnostic) and DNaseI (Sigma-Aldrich). The organs were then smashed through a $40-\mu \mathrm{m}$ cell strainer (BD Biosciences, Franklin Lakes, NJ). Single-cell suspensions were then stained for flow cytometry or stimulated for $4 \mathrm{~h}$ in the presence of $50 \mathrm{ng} \mathrm{ml}^{-1}$ phorbol 12 -myristate 13 -acetate (Sigma-Aldrich) and $750 \mathrm{ng} \mathrm{ml}^{-1}$ ionomycin (Invitrogen) with $10 \mu \mathrm{g} \mathrm{ml}^{-1}$ Brefeldin A (Sigma-Aldrich) or $2 \mu \mathrm{M}$ Monensin (Sigma-Aldrich).

Flow cytometry. Antibodies and corresponding clones used for flow cytometry were as follows: IFN $\gamma$ fluorescein isothiocyanate (FITC) or allophycocyanin (APC; XMG1.2), IL-17A phycoerythrin (PE; TC1118H10), CD4 Pacific blue (GK1.5), CD8 PE-cy7 (53-6.7), CD3 APCcy7 (145-2C11), CD11b PerCp-cy5.5 (M1/70), CD11c FITC (N418), MHC II APC-cy7 (M5/114.15.2), F4/80 APC (BM8), CD103 biotin (M290 or 2E7), CD25 PerCp-Cy5.5 (PC61), Thy1.2 biotin (30-H12), CD40 FITC (HM40-3), CD80 PE (16-10A1), CD86 PerCp-cy5.5 (GL-1) CD45.2 APC (104) and streptavidin-APC were purchased from BD Biosciences or Biolegend (San Diego, CA). Foxp3 AlexaFluor700 (FJK-16a, eBioscience, San Diego, CA) and Helios FITC (22F6, Biolegend) were used in combination with the Foxp3 staining kit (eBioscience). Data were acquired on a Cyan (Beckman Coulter, Nyon, Switzerland) or on an LSRII (BD Biosciences) and analyzed with FlowJo software (Tree Star, Ashland, OR).

Cytokine ELISA. To measure IL-12p40, goat anti-mouse antibody (clone C15.6) was used for capture and biotin-conjugated antibody (clone C17.8) followed by alkaline-phosphatase conjugated streptavidin for detection. Recombinant mouse IL-12 was used as standard. All antibodies were purchased from eBioscience.

Histology and immunofluorescence. The colon was prepared using the "Swiss roll" technique ${ }^{46}$ to evaluate the entire longitudinal section on one slide. For histological evaluation, tissues were fixed in $4 \%$ paraformaldehyde, embedded in paraffin, sectioned at $4 \mu \mathrm{m}$, stained with haematoxylin and eosin, and examined histologically. For immunofluorescence, tissues were embedded in optimal cutting temperature compound (Tissue-Tek, Sakura Finetek Europe B.V., Alphen aan den Rijn, The Netherlands) and snap frozen in a bath of 
isopentane on dry ice. In all, $8-\mu \mathrm{m}$ sections were prepared and stained with IgA FITC (c10-3, BD Bioscience), CD11c PE (N418, eBioscience), B220 alexa 647 (RA3-6B2, Biolegend), and DAPI (Sigma-Aldrich). Lymphoid follicle maturation was assessed depending on the presence of B220 ${ }^{+}$cells (low to none: immature follicles, high: mature follicles); size was assessed by visual observation using a Zeiss Axioplan microscope (Carl Zeiss Inc, Thornwood, NJ).

T-cell transfer. Total CD4 ${ }^{+}$cells were isolated from the spleen of wildtype CD45.1 or TSLPR ${ }^{-1-}$ with anti-CD4 MACS beads (Miltenyi Biotec, Bergisch Gladbach, Germany). A total of $4 \times 10^{6}$ wild-type and TSLPR $^{-/-}$cells (1:1 ratio) were transferred to germ-free RAG ${ }^{-1-}$ recipients. ASF colonization was started on the day of T-cell transfer and recipient mice were analyzed after 28 days.

Statistics. Results represent mean \pm SEM. Statistical significance was determined by the Student's $t$-test $\left({ }^{\star} P<0.05,{ }^{* \star} P<0.01,{ }^{\star * \star} P<0.001\right)$.

SUPPLEMENTARY MATERIAL is linked to the online version of the paper at http://www.nature.com/mi

\section{ACKNOWLEDGEMENTS}

We thank the École Polytechnique Fédérale de Lausanne animal facility as well as the Clean mouse facility of the University of Bern, Jessica Dessimoz and the École Polytechnique Fédérale de Lausanne histology core facility for histological sectioning and staining. Miguel Garcia and the École Polytechnique Fédérale de Lausanne flow cytometry core facility. I.M. and N.L.H. are supported by the Swiss Vaccine Research Institute. M.B.G. is supported by an SNF Ambizione fellowship and K.D.M. is supported by an SNF and an ERC starting grant.

\section{DISCLOSURE}

The authors declared no conflict of interest.

c) 2013 Society for Mucosal Immunology

\section{REFERENCES}

1. Barnes, M.J. \& Powrie, F. Regulatory T cells reinforce intestinal homeostasis. Immunity 31, 401-411 (2009).

2. Geuking, M.B., McCoy, K.D. \& Macpherson, A.J. The continuum of intestinal CD4 + T cell adaptations in host-microbial mutualism. Gut Microbes 2, 353-357 (2011).

3. Geuking, M.B. et al. Intestinal bacterial colonization induces mutualistic regulatory T cell responses. Immunity 34, 794-806 (2011).

4. Atarashi, K. et al. Induction of colonic regulatory T cells by indigenous Clostridium species. Science 331, 337-341 (2011).

5. Macpherson, A.J., Geuking, M.B. \& McCoy, K.D. Innate and adaptive immunity in host-microbiota mutualism. Front. Biosci. (Schol. Ed.) 4, 685-698 (2012).

6. Friend, S.L. et al. A thymic stromal cell line supports in vitro development of surface lgM + B cells and produces a novel growth factor affecting $B$ and $T$ lineage cells. Exp. Hematol. 22, 321-328 (1994).

7. Al-Shami, A. et al. A role for thymic stromal lymphopoietin in CD4(+) Tcell development. J. Exp. Med. 200, 159-168 (2004).

8. Ziegler, S.F. \& Artis, D. Sensing the outside world: TSLP regulates barrier immunity. Nat. Immunol. 11, 289-293 (2010).

9. He, R. \& Geha, R.S. Thymic stromal lymphopoietin. Ann. NY Acad. Sci. 1183, 13-24 (2010).

10. Spadoni, I., lliev, I.D., Rossi, G. \& Rescigno, M. Dendritic cells produce TSLP that limits the differentiation of Th17 cells, fosters Treg development, and protects against colitis. Mucosal Immuno./ 5, 184-193 (2012).

11. Taylor, B.C. et al. TSLP regulates intestinal immunity and inflammation in mouse models of helminth infection and colitis. J. Exp. Med. 206, 655-667 (2009).

12. Zeuthen, L.H., Fink, L.N. \& Frokiaer, H. Epithelial cells prime the immune response to an array of gut-derived commensals towards a tolerogenic phenotype through distinct actions of thymic stromal lymphopoietin and transforming growth factor-beta. Immunology 123, 197-208 (2008).
13. Allakhverdi, Z. et al. Thymic stromal lymphopoietin is released by human epithelial cells in response to microbes, trauma, or inflammation and potently activates mast cells. J. Exp. Med. 204, 253-258 (2007).

14. Kato, A., Favoreto, S. Jr, Avila, P.C. \& Schleimer, R.P. TLR3- and Th2 cytokine-dependent production of thymic stromal lymphopoietin in human airway epithelial cells. J. Immunol. 179, 1080-1087 (2007).

15. Lee, H.C. \& Ziegler, S.F. Inducible expression of the proallergic cytokine thymic stromal lymphopoietin in airway epithelial cells is controlled by NFkappaB. Proc. Natl. Acad. Sci. USA 104, 914-919 (2007).

16. Humphreys, N.E., Xu, D., Hepworth, M.R., Liew, F.Y. \& Grencis, R.K. IL-33, a potent inducer of adaptive immunity to intestinal nematodes. J. Immunol. 180, 2443-2449 (2008).

17. Ito, T. et al. TSLP-activated dendritic cells induce an inflammatory Thelper type 2 cell response through OX40 ligand. J. Exp. Med. 202, 1213-1223 (2005).

18. Soumelis, V. et al. Human epithelial cells trigger dendritic cell mediated allergic inflammation by producing TSLP. Nat. Immunol. 3, 673-680 (2002).

19. Ying, S. et al. Thymic stromal lymphopoietin expression is increased in asthmatic airways and correlates with expression of Th2-attracting chemokines and disease severity. J. Immunol. 174, 8183-8190 (2005).

20. He, B. et al. Intestinal bacteria trigger T cell-independent immunoglobulin A(2) class switching by inducing epithelial-cell secretion of the cytokine APRIL. Immunity 26, 812-826 (2007).

21. Hanabuchi, S. et al. Thymic stromal lymphopoietin-activated plasmacytoid dendritic cells induce the generation of FOXP3 + regulatory T cells in human thymus. J. Immunol. 184, 2999-3007 (2010).

22. Siracusa, M.C. et al. TSLP promotes interleukin-3-independent basophil haematopoiesis and type 2 inflammation. Nature 477, 229-233 (2011).

23. Massacand, J.C. et al. Helminth products bypass the need for TSLP in Th2 immune responses by directly modulating dendritic cell function. Proc. Natl. Acad. Sci. USA 106, 13968-13973 (2009).

24. Reardon, C. et al. Thymic stromal lymphopoetin-induced expression of the endogenous inhibitory enzyme SLPI mediates recovery from colonic inflammation. Immunity 35, 223-235 (2011).

25. Dewhirst, F.E. et al. Phylogeny of the defined murine microbiota: altered Schaedler flora. Appl. Environ. Microbiol. 65, 3287-3292 (1999).

26. Rivollier, A., He, J., Kole, A., Valatas, V. \& Kelsall, B.L. Inflammation switches the differentiation program of Ly6Chi monocytes from antiinflammatory macrophages to inflammatory dendritic cells in the colon. J. Exp. Med. 209, 139-155 (2012).

27. Pabst, O. \& Bernhardt, G. The puzzle of intestinal lamina propria dendritic cells and macrophages. Eur. J. Immunol. 40, 2107-2111 (2010).

28. Schulz, O. et al. Intestinal CD103 + , but not CX3CR1 + , antigen sampling cells migrate in lymph and serve classical dendritic cell functions. J. Exp. Med. 206, 3101-3114 (2009).

29. Bogunovic, M. et al. Origin of the lamina propria dendritic cell network. Immunity 31, 513-525 (2009).

30. Denning, T.L., Wang, Y.C., Patel, S.R., Williams, I.R. \& Pulendran, B. Lamina propria macrophages and dendritic cells differentially induce regulatory and interleukin 17-producing Tcell responses. Nat. Immunol. 8, 1086-1094 (2007)

31. McGovern, D. \& Powrie, F. The IL23 axis plays a key role in the pathogenesis of IBD. Gut 56, 1333-1336 (2007).

32. Rimoldi, M. et al. Intestinal immune homeostasis is regulated by the crosstalk between epithelial cells and dendritic cells. Nat. Immunol. 6, 507-514 (2005).

33. Bouskra, D. et al. Lymphoid tissue genesis induced by commensals through NOD1 regulates intestinal homeostasis. Nature 456, 507-510 (2008).

34. Lochner, M. et al. Microbiota-induced tertiary lymphoid tissues aggravate inflammatory disease in the absence of RORgamma t and LTi cells. J. Exp. Med. 208, 125-134 (2010).

35. Fagarasan, S. et al. Critical roles of activation-induced cytidine deaminase in the homeostasis of gut flora. Science 298, 1424-1427 (2002).

36. Lorenz, R.G., Chaplin, D.D., McDonald, K.G., McDonough, J.S. \& Newberry, R.D. Isolated Iymphoid follicle formation is inducible and dependent upon lymphotoxin-sufficient $B$ lymphocytes, lymphotoxin beta receptor, and TNF receptor I function. J. Immunol. 170, 5475-5482 (2003). 
37. Thornton, A.M. et al. Expression of Helios, an Ikaros transcription factor family member, differentiates thymic-derived from peripherally induced Foxp3 + T regulatory cells. J. Immunol. 184, 3433-3441.

38. Lathrop, S. et al. Peripheral education of the immune system by colonic commensal microbiota. Nature 478, 250-254.

39. Mileti, E., Matteoli, G., lliev, I.D. \& Rescigno, M. Comparison of the immunomodulatory properties of three probiotic strains of Lactobacilli using complex culture systems: prediction for in vivo efficacy. PLoS One 4, e70562009).

40. Newberry, R.D. Intestinal lymphoid tissues: is variety an asset or a liability? Curr. Opin. Gastroenterol. 24, 121-128 (2008).

41. Rangel-Moreno, J. et al. The development of inducible bronchusassociated lymphoid tissue depends on IL-17. Nat. Immunol. 12, 639646 (2011).
42. Iliev, I.D. et al. Human intestinal epithelial cells promote the differentiation of tolerogenic dendritic cells. Gut 58, 1481-1489 (2009).

43. Carpino, N. et al. Absence of an essential role for thymic stromal lymphopoietin receptor in murine B-cell development. Mol. Cell Biol. 24, 2584-2592 (2004).

44. Kang, Z. et al. Epithelial cell-specific Act1 adaptor mediates interleukin25-dependent helminth expulsion through expansion of Lin $(-) \mathrm{c}-\mathrm{Kit}(+)$ innate cell population. Immunity 36, 821-833 (2012).

45. Lutz, M.B. et al. An advanced culture method for generating large quantities of highly pure dendritic cells from mouse bone marrow. J. Immunol. Methods 223, 77-92 (1999).

46. Moolenbeek, C. \& Ruitenberg, E.J. The 'Swiss roll': a simple technique for histological studies of the rodent intestine. Lab. Anim. 15, 57-59 (1981). 